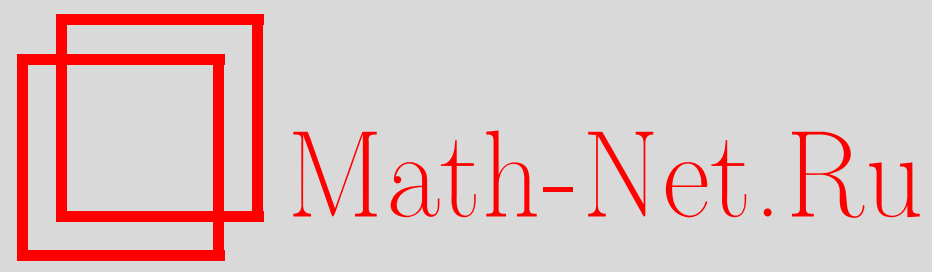

А. А. Борисенко, О слоениях отрицательной внешней кривизны на компактных римановых многообразиях, Матем. заметки, 1997, том 62, выпуск 5, 673-676

DOI: https://doi.org/10.4213/mzm1653

Использование Общероссийского математического портала Math-Net.Ru подразумевает, что вы прочитали и согласны с пользовательским соглашением http://www . mathnet.ru/rus/agreement

Параметры загрузки:

IP: 54.92 .164 .108

26 апреля 2023 г., $18: 21: 41$ 
УДК 514

\title{
О СЛОЕНИЯХ ОТРИЦАТЕЛЬНОЙ ВНЕШНЕЙ КРИВИЗНЫ НА КОМПАКТНЫХ РИМАНОВЫХ МНОГООБРАЗИЯХ
}

\section{А. А. Борисенко}

\begin{abstract}
В заметке изучается топологическое строение компактных римановых многообразий, на которых существуют гиперболические слоения.

Библиография: 4 названия.
\end{abstract}

В заметке изучается топологическоестроение компактных римановых многообразий, на которых существуют гиперболические слоения.

ОПРЕДЕЛЕниЕ. Слоениена римановом многообразии называется гиперболическим, если двумерная секционная кривизна слоя меньше двумерной секционной кривизны объемлющего пространства вдоль площадок, касательных к слою.

Если размерность слоя равна $l$, то минимальная размерность объемлющего пространства, когда может существовать гиперболическое слоение, равна $2 l-1$. Имеет место

ТЕОремА. Пусть $F^{l}$ - гиперболическое слоение на односвязном компактном римановом многообразии $M^{2 l-1}$. Eсли $l=2,3,4$, то многообразие $M^{2 l-1}$ параллелизуемое.

ПримечАниЕ. По нашему мнению теорема верна при любых значениях $l \geqslant 2$.

Пусть задана система квадратных уравнений

$$
A^{\alpha}(X, X)=A_{i j}^{\alpha} x^{i} x^{j}=0, \quad i, j=1, \ldots, l, \quad \alpha=1, \ldots, N
$$

где $X$ есть вектор с координатами $x^{1}, \ldots, x^{l}$. Так как система (1) однородна, решения, отличающиеся друг от друга ненулевым множителем, различать не будем. Пусть $A(X, Y)$ есть вектор с координатами $A_{i j}^{\alpha} x^{i} y^{j}$, где $\langle\cdot, \cdot\rangle$ - скалярное произведение векторов в касательном пространстве в точке $M^{2 l-1}$.

ЛЕмма. Если в системе уравнений (1) $N=l-1$ и для любых неколлинеарных векторов $X, Y$ выполняется условие

$$
\langle A(X, X), A(Y, Y)\rangle-\langle A(X, Y), A(X, Y)\rangle<0
$$

то система имеет $2^{l-1}$ действительных нетривиальных решений, среди которых найдется $l$ линейно независимых решений. Любые три решения не лежат в одной плоскости, в трехмерном подпространстве не мохет лехсать более четырех решений. 
ДокАЗАтЕльство. Эта лемма обобщает лемму, доказанную Т. Отзуки [1]. Сначала покажем, что все решения системы (1) с точностью до комплексного множителя действительны. Пусть $Z_{0}=X_{0}+i Y_{0}-$ комплексное решение системы (1). Тогда

$$
A\left(Z_{0}, Z_{0}\right)=A\left(X_{0}, X_{0}\right)-A\left(Y_{0}, Y_{0}\right)+2 i A\left(X_{0}, Y_{0}\right)=0 \text {. }
$$

Отсюда $A\left(X_{0}, X_{0}\right)=A\left(Y_{0}, Y_{0}\right), A\left(X_{0}, Y_{0}\right)=0$. Если $X_{0} \neq \lambda Y_{0}$, то

$$
\left\langle A\left(X_{0}, X_{0}\right), A\left(Y_{0}, Y_{0}\right)\right\rangle-\left\langle A\left(X_{0}, Y_{0}\right), A\left(X_{0}, Y_{0}\right)\right\rangle>0 .
$$

Это противоречит неравенству (2).

Пусть $t_{0}=\left(t_{0}^{1}, \ldots, t_{0}^{l}\right)$ - единичное решение системы $(1)$. Оно удовлетворяет уравнениям

$$
A^{\alpha}=A_{i j}^{\alpha} t^{i} t^{j}=0, \quad A^{l}=\langle t, t\rangle=1 .
$$

Матрица Якоби системы (3) при $t=t_{0}$ имеет вид

$$
\frac{\partial A^{\alpha}}{\partial t^{k}}=2 A_{i k}^{\alpha} t_{0}^{i}, \quad \alpha=1, \ldots, l-1, \quad \frac{\partial A^{l}}{\partial t^{k}}=2 t_{0}^{k}, \quad k=1, \ldots, l .
$$

Допустим что якобиан равен 0 . Тогда существует ненулевой вектор $\lambda=\left(\lambda^{1}, \ldots, \lambda^{l}\right)$ такой, что

$$
A_{i k}^{\alpha} t_{0}^{i} \lambda^{k}=A^{\alpha}\left(t_{0}, \lambda\right)=0, \quad\left\langle t^{0}, \lambda\right\rangle=0 .
$$

Отсюда следует, что для ортогональных векторов $t_{0}, \lambda$ имеет место равенство

$$
\left\langle A\left(t_{0}, t_{0}\right), A(\lambda, \lambda)\right\rangle-\left\langle A\left(t_{0}, \lambda\right), A\left(t_{0}, \lambda\right)\right\rangle=0 .
$$

Это противоречит неравенству (2). Значит, якобиан системы (3) при $t=t_{0}$ отличен от 0. Отсюда следует, что в точке $t=t_{0}$ гиперповерхности $A^{\alpha}(t, t)=0$ пересекаются трансверсально, поэтому $t=t_{0}$ является изолированным решением системы $(1)$, кратность которого равна 1. Теорема Безу [2, с. 268] утверждает, что число решений системы $l-1$ уравнений $c l$ неизвестными или бесконечно, или равно произведению степеней уравнений, если решения считать с их кратностью. Так как решения системы (1) изолированы и действительны, система имеет $2^{l-1}$ различных действительных решений. Докажем, что среди решений найдется $l$ линейно независимых. Допустим противное, пусть все решения системы (1) лежат в плоскости $x^{l}=0$. На плоскости $x^{l}=1$ рассмотрим функцию $f=\langle A(X, X), A(X, X)\rangle$. Каждый вектор с концом в этой плоскости имеет вид $X=\mu X^{0}+e$, где $X^{0}-$ единичньй вектор в плоскости $x^{l}=0, e-$ единичный вектор нормали к этой плоскости. Тогда

$$
A(X, X)=\mu^{2} A\left(X_{0}, X_{0}\right)+\mu A\left(X_{0}, e\right)+A(e, e) .
$$

Если $A\left(X_{0}, X_{0}\right) \neq 0$, то при $\mu \rightarrow \infty$ функция $f$ на луче с направлением $X_{0}$ имеет порядок $\mu^{4}$ и также стремится к бесконечности. Если $A\left(X_{0}, X_{0}\right)=0$, то из (2) следует, что $A\left(X_{0}, e\right) \neq 0$, и функция $f$ имеет на этом луче порядок $\mu^{2}$. Отсюда следует, что при $\left\langle X_{0}, X_{0}\right\rangle \rightarrow \infty$ функция $f \rightarrow \infty$. Поэтому найдется такое значение $X=Y_{0}$, при котором функция $f$ принимает наименьшее значение, отличное от 0 . Пусть $F=f-\mu\left(x^{l}\right)^{2}$. В точке минимума

$$
\begin{gathered}
\frac{\partial F}{\partial x^{i}}=2 \sum_{\alpha=1}^{l-1} A_{s j}^{\alpha} y_{0}^{s} y_{0}^{j} A_{i j}^{\alpha} y_{0}^{j}-\mu \delta_{i}^{l} y_{0}^{l}=0 \\
\frac{\partial F}{\partial x^{i} \partial x^{j}}=2 \sum_{\alpha=1}^{l-1} A_{s t}^{\alpha} y_{0}^{s} y_{0}^{t} A_{i j}^{\alpha}+4 \sum_{\alpha=1}^{l-1} A_{i t}^{\alpha} y_{0}^{t} A_{s j}^{\alpha} y_{0}^{s}-\mu \delta_{i}^{l} \delta_{j}^{l} .
\end{gathered}
$$


Из необходимого условия минимума следует, что

$$
\left.\left\langle A\left(Y_{0}, Y_{0}\right), A\left(Y_{0}, t\right)\right\rangle=0, \quad 2\left\langle A\left(Y_{0}, Y_{0}\right), A(t, t)\right\rangle+4\left\langle A\left(Y_{0}, t\right), A Y_{0}, t\right)\right\rangle \geqslant 0 .
$$

Для $t=t_{0} A\left(t_{0}, t_{0}\right)=0$. Поэтому из (4) следует, что

$$
\left\langle A\left(Y_{0}, t_{0}\right), A\left(Y_{0}, t_{0}\right)\right\rangle \geqslant 0 \text {. }
$$

Это противоречит неравенству (2). Поэтому предположение, что все решения системы (1) лежат в плоскости меньшей размерности, неверно. Любые три решения системы (1) линейно независимы. Пусть $t_{1}, t_{2}$ - непропорциональные решения системы (1), $t=\lambda t_{1}+\lambda t_{2}-$ любой вектор из плоскости, натянутой на эти векторы. Тогда

$$
A(t, t)=2 \lambda_{1} \lambda_{2} A\left(t_{1}, t_{2}\right) .
$$

Из $(2)$ следует, что $A\left(t_{1}, t_{2}\right) \neq 0$. Поэтому в плоскости нет других решений системы. В трехмерной плоскости $E^{3}$ лежит не более четырех различных решений системы (1). Действительно, пусть $t_{1}, t_{2}, t_{3}$ - три различных решения системы (1). Пусть $t=\lambda^{1} t_{1}+\lambda^{2} t_{2}+\lambda^{3} t_{3}$. Тогда

$$
A(t, t)=\lambda^{i} \lambda^{j} A\left(t_{i}, t_{j}\right), \quad i \neq j, \quad i<j, \quad i, j=1,2,3 .
$$

Из $(2)$ следует, что векторы $A\left(t_{i}, t_{j}\right)$ неколлинеарны, в крайнем случае они могут лежать в одной плоскости. Если $t$ - решение системы (1), то мы имеем этот случай. С точностью до коэффициента пропорциональности существует единственный набор чисел $c_{1}, c_{2}, c_{3}$ таких, что

$$
c_{1} A\left(t_{2}, t_{2}\right)+c_{2} A\left(t_{3}, t_{1}\right)+c_{3} A\left(t_{1}, t_{2}\right)=0 .
$$

Поэтому

$$
\lambda^{1} \lambda^{2}=\rho c_{3}, \quad \lambda^{3} \lambda^{1}=\rho c_{2}, \quad \lambda^{2} \lambda^{3}=\rho c_{1} .
$$

Мы получаем единственное с точностью до пропорциональности решение.

ДоКАЗАТЕЛЬСТВо ТЕОРЕМЫ. Из формулы Гаусса [3] следует, что

$$
\gamma_{2 / F}=\gamma_{2 / M}+\langle A(X, X), A(Y, Y)\rangle-\langle A(X, Y), A(X, Y)\rangle,
$$

где $\gamma_{2 / F}, \gamma_{2 / M}$ - двумерные секционные кривизны слоя $F^{l}$ и объемлющего пространства $M^{2 l-1}$ в направлении двумерной площадки, натянутой на векторы $X, Y$, касательные к слою. $A(X, X)$ - вектор с координатами $A_{i j}^{\alpha} x^{i} x^{j}$, где $A_{i j}^{\alpha}$ - коэффициенты вторых квадратичных форм слоя относительно ортонормированного базиса нормалей $n_{\alpha}, X$, $Y$ - взаимно ортогональные единичные векторы, касательные к слою. Из гиперболичности слоения следует, что

$$
\langle A(X, X), A(Y, Y)\rangle-\langle A(X, Y), A(X, Y)\rangle<0
$$

и система квадратных уравнений

$$
A_{i j}^{\alpha} t^{i} t^{j}=0, \quad\langle t, t\rangle=0
$$


в каждой точке слоя удовлетворяет условию леммы 1 . Ненулевые решения этой системы определяют асимптотические направления на слоях. Из теоремы о неявной функции и невырожденности якобиана из решений системы (3) следует, что локально асимптотические направления распадаются на $2^{l}$ регулярных единичных векторных поля $V_{i}$, заданных на многообразии $M^{2 l-1}$. Пусть $T M^{2 l-1}$ - касательное расслоение многообразия $M^{2 l-1}$. $M_{1}$ - совокупность единичных асимптотических векторов к слоям. Так как локально они распадаются на регулярные векторные поля, $M_{1}$ является компактным дифференцируемым многообразием [4]. Пусть $\imath: M_{1} \rightarrow T M^{2 l-1}$ - отображение включения, $\varphi: T M^{2 l-1} \rightarrow M^{2 l-1}-$ естественная проекция. Тогда отображение $\varphi$ ○ $\imath: M_{1} \rightarrow M^{2 l-1}$ является локальным диффеоморфизмом, а компактное многообразие $M_{1}$ - накрывающей многообразия $M^{2 l-1}$ со слоем конечной мощности. Так как $M^{2 l-1}$ односвязно, $M_{1}$ состоит из связных компонент, каждая из которых диффеоморфна $M^{2 l-1}$. Каждая компонента есть глобальное невырожденное асимптотическое векторное поле, касательное к слою, определенное на $M^{2 l-1}$. При $l=2$ утверждение очевидно. Пусть $l=3$ и $V_{1}$ - глобальное невырожденное асимптотическое векторное поле на $M^{5}, V_{2}, V_{3}$ - два других асимптотических векторных поля. Из леммы следует, что $V_{1}, V_{2}, V_{3}$ - линейно независимые векторные поля. Пусть $\widetilde{V}_{2}, \widetilde{V}_{3}$ - ортогональные к $V_{1}$ единичные векторные поля, направление которых совпадает с ортогональной проекцией полей $V_{2}, V_{3}$ в касательном пространстве к слою на плоскость, перпендикулярную к $V_{1}$. Пусть $v=\left(v_{1}, \ldots, v_{l}\right)$ - значение векторного поля $V_{1}$ в точке $Q$. Тогда $A_{i j}^{\alpha} v^{i} v^{j}=0$ и $\langle A(X, v), A(X, v)\rangle>0$ для векторов $X$, ортогональных $v$. Отображение $A: T_{Q}^{\perp} \rightarrow N_{Q}$ ортогонального дополнения $T_{Q}^{\perp}$ вектора $v$ в касательном пространстве к слою на нормальное пространство к слою $N_{Q}$ является изоморфизмом. Это отображение ставит в соответствие каждому вектору $X \in T_{Q}^{\perp}(v)$ вектор с координатами $A_{i j}^{\alpha} X^{i} v^{j}$. При этом векторные поля $\widetilde{V}_{2}, \widetilde{V}_{3}$ отображаются в нормальные линейно независимые поля $A\left(\widetilde{V}_{2}\right), A\left(\widetilde{V}_{3}\right)$. Векторные поля $V_{1}, V_{2}, V_{3}, A\left(\widetilde{V}_{2}\right), A\left(\widetilde{V}_{3}\right)$ линейно независимы в каждой точке. Отсюда следует, что $M^{5}$ - параллелизуемое многообразие. Пусть $l=4$. Аналогично случаю $l=3$ асимптотические векторные поля $V_{1}, V_{2}, V_{3}$ линейно независимы; $V_{4}$ - поле направлений, ортогональное векторным полям $V_{1}, V_{2}, V_{3}$ и касательное к слоям. В силу односвязности многообразия $M^{7}$ мы можем выбрать единичное глобальное векторное поле $V^{4}$. В дальнейшем ход доказательства совпадает со случаeм $l=3$.

\section{СПИСОК ЦИТИРОВАННОЙ ЛИТЕРАТУРЫ}

[1] Otsuki T. On the existence of solution of quadratic equations and its geometrical application // Proc. Japan Acad. Ser. A. Math. Sci. 1953. V. 29. P. 99-100.

[2] Шафаревич И. Р. Основы алгебраической геометрии. М.: Наука, 1972.

[3] Эйзенхарт Л. Риманова геометрия. М.: ИЛ, 1948.

[4] Борисенко А. А. О компактных поверхностях отрицательной внешней кривизны в римановом пространстве // Укр. геом. сб. 1976. Т. 19. С. 9-11.

Харьковский государственный университет

Поступило

E-mail: Alexander.A.Borisenko@univer.kharkov.ua

Исправленный вариант 